\title{
Transnasal odontoid resection: is there an anatomic explanation for differing swallowing outcomes?
}

\author{
Kathryn M. Van Abel, M.D., ${ }^{1}$ Grant W. Mallory, M.D., ${ }^{2}$ Jan L. Kasperbauer, M.D., ${ }^{1}$ \\ Eric J. Moore, M.D. ${ }^{1}$ Daniel L. Price, M.D. ${ }^{1}$ Erin K. O’Brien, M.D. ${ }^{1}$ \\ Kerry D. Olsen, M.D., ${ }^{1}$ William E. Krauss, M.D., ${ }^{2}$ Michelle J. Clarke, M.D., ${ }^{2}$ \\ Mark E. Jentoft, M.D., ${ }^{3}$ AND Jamie J. VAN Gompel, M.D. ${ }^{2}$
}

${ }^{1}$ Division of Otolaryngology Head and Neck Surgery, and Departments of ${ }^{2}$ Neurologic Surgery and ${ }^{3}$ Anatomic Pathology, Mayo Clinic, Rochester, Minnesota

\begin{abstract}
Object. Swallowing dysfunction is common following transoral (TO) odontoidectomy. Preliminary experience with newer endoscopic transnasal (TN) approaches suggests that dysphagia may be reduced with this alternative. However, the reasons for this are unclear. The authors hypothesized that the TN approach results in less disruption of the pharyngeal plexus and anatomical structures associated with swallowing. The authors investigate the histological and gross surgical anatomical relationship between pharyngeal plexus innervation of the upper aerodigestive tract and the surgical approaches used (TN and TO). They also review the TN literature to evaluate swallowing outcomes following this approach.

Methods. Seven cadaveric specimens were used for histological $(n=3)$ and gross anatomical $(n=4)$ examination of the pharyngeal plexus with the TO and TN surgical approaches. Particular attention was given to identifying the location of cranial nerves (CNs) IX and X and the sympathetic chain and their contributions to the pharyngeal plexus. S100 staining was performed to assess for the presence of neural tissue in proximity to the midline, and fiber density counts were performed within $1 \mathrm{~cm}$ of midline. The relationship between the pharyngeal plexus, clivus, and upper cervical spine (C1-3) was defined.

Results. Histological analysis revealed the presence of pharyngeal plexus fibers in the midline and a significant reduction in paramedian fiber density from $\mathrm{C}-2$ to the lower clivus $(\mathrm{p}<0.001)$. None of these paramedian fibers, however, could be visualized with gross inspection or layer-by-layer dissection. Laterally based primary pharyngeal plexus nerves were identified by tracing their origins from CNs IX and X and the sympathetic chain at the skull base and following them to the pharyngeal musculature. In addition, the authors found 15 studies presenting 52 patients undergoing TN odontoidectomy. Of these patients, only 48 had been swallowing preoperatively. When looking only at this population, 83\% (40 of 48) were swallowing by Day 3 and 92\% (44 of 48) were swallowing by Day 7.

Conclusions. Despite the midline approach, both TO and TN approaches may injure a portion of the pharyngeal plexus. By limiting the TN incision to above the palatal plane, the surgeon avoids the high-density neural plexus found in the oropharyngeal wall and limits injury to oropharyngeal musculature involved in swallowing. This may explain the decreased incidence of postoperative dysphagia seen in TN approaches. However, further clinical investigation is warranted.
\end{abstract}

(http://thejns.org/doi/abs/10.3171/2014.7.FOCUS14338)

KEY Words • endoscopic $\quad$ transnasal $\bullet$ odontoidectomy $\bullet$ dysphagia
pharyngeal plexus

A NTERIOR cervical spine surgery, including anterior decompressive odontoidectomy, has traditionally been associated with a relatively high risk for postoperative dysphagia., 2,3,6,26 This complication has been estimated to impact up to $60 \%$ of patients following anterior cervical spine surgery when tested carefully. $., 20,30$ The etiology for postoperative dysphagia is likely multifactorial, but contributing factors may include edema and thickening of the posterior pharyngeal wall, injury to the pharyngeal plexus, recurrent or superior laryngeal nerve

Abbreviations used in this paper: $\mathrm{CN}=$ cranial nerve; $\mathrm{TN}=$ transnasal; $\mathrm{TO}=$ transoral. injury, preoperative debility, and neuromuscular scarring and fibrosis. ${ }^{20}$ While generally transient ( $<6$ months), dysphagia can result in malnutrition, dehydration, aspiration, anxiety, depression, isolation, and significant negative social, emotional, and socioeconomic outcomes. ${ }^{4}$

Currently, odontoid resection can be accomplished through several different surgical approaches, including transnasal (TN) and transoral (TO) approaches. Often the anatomy dictates which approach is appropriate for the removal of the odontoid. However, the best approach, indications, and potential risks for each remain controversial. Recently, several authors have proposed that the TN approach may decrease the risk of postoperative dyspha- 
gia compared with the traditional TO approach. ${ }^{6,29}$ The question is: Why? While there may be many overlapping factors, as described above, understanding how the neuromuscular anatomy and associated physiology relate to the surgical anatomy is crucial to better predict the risk for postoperative dysphagia for these varying techniques.

We therefore set out to investigate the relationship between the neuromuscular anatomy of the upper aerodigestive tract and the surgical approaches used for both TN and TO odontoidectomy. We also conducted a review of the TN literature to identify cases in which swallowing outcomes were reported to better understand the risk compared with more traditional approaches.

\section{Methods}

\section{Anatomical Study}

Our institution's biospecimens committee reviewed our proposal and approved the use of 7 fresh-frozen head and neck specimens ( 6 head and neck only and 1 head, neck, and torso). These specimens were frozen intact immediately after donation.

\section{Histological Analysis}

Three specimens were processed to appropriate sections using a band saw. As shown in Fig. 1, the specimens were cut parallel to the lateral segments of the vertebral body taking care to maintain midline, with a goal of 1 $\mathrm{cm}$ of tissue lateral to midline in both directions. Once the initial specimen block had been prepared, the following tangential cuts were made: $\mathrm{C} 3-4$ disc space, $\mathrm{C}-3$ midbody, C2-3 disc space, C-2 body, C1-2 interface, C-1 midtubercle, $\mathrm{C}-1$ top (tip of odontoid), inferior clivus, and

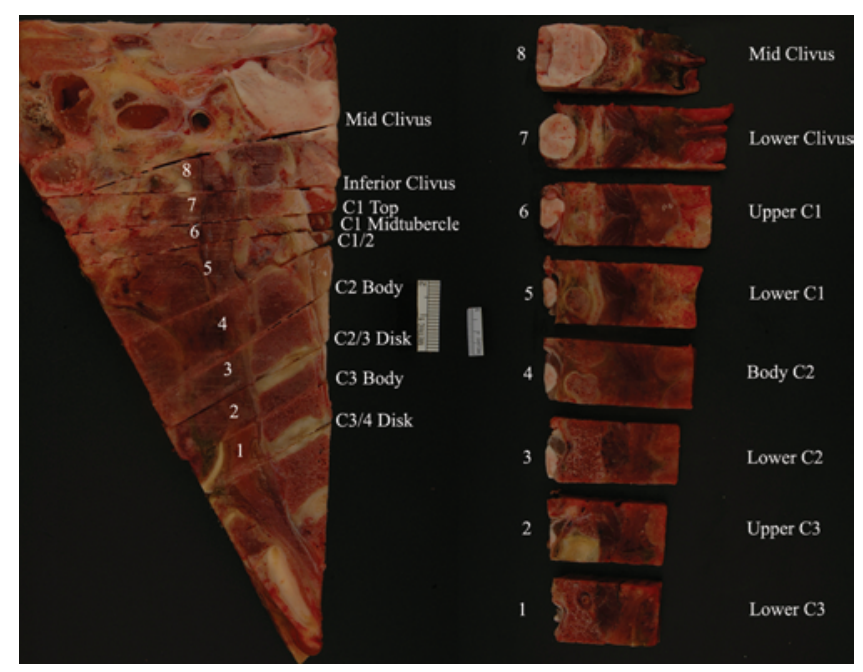

Fig. 1. Gross sections of the cadaveric pharynx as used for histological and nerve density analysis for 3 specimens. Two-centimeter-wide sagittal view of the reassembled pharynx and associated structures from which the blocks were cut (left). The 3 specimens used for histological analysis of neural density were all cut in this manner. Axial view of the separated blocks corresponding to their levels as numbered (right). Blocks were numbered 1-8 with the corresponding anatomical level labeled (Level 1, lower C-3; Level 2, upper C-3; Level 3, lower C-2; Level 4, body C-2; Level 5, lower C-1; Level 6, upper C-1; Level 7, lower clivus; and Level 8, midclivus). superior clivus. These cuts produced the following segments for histological analysis (Fig. 1): lower C-3 (Level 1), upper C-3 (Level 2), lower C-2 (Level 3), body C-2 (Level 4), lower C-1 (Level 5), upper C-1 (Level 6), lower clivus (Level 7), and upper clivus (Level 8). Once these blocks were made, the periosteum of the anterior vertebral body or the clivus was separated and the anterior soft tissues were cataloged, cartridged, and immediately fixed in formalin.

From this tissue, $\mathrm{H} \& \mathrm{E}$ and $\mathrm{S} 100$ immunohistochemical staining was performed on each level (Fig. 2). Nerve counts were performed on the posterior pharynx from the S100-stained slides and were determined based off of an area $2 \mathrm{~cm}$ wide, centered on midline, and included all of the soft tissue from the mucosa posterior to where the tissue meets the vertebral column. Microscopically, the midline was determined based on the raphae, or the point at which the pharyngeal muscles met in the midline. In one of the 3 cadavers, the dissection was too medial on one side and adequate counts could only be made $1 \mathrm{~cm}$ from midline on the contralateral side. The counts at each level were determined by the number of S100-positive nerves present. If a single nerve followed a serpiginous course and was sectioned multiple times within 1 level, it was counted each time it appeared on the slide as a separate nerve section. Scanning for nerves was done using the $10 \times$ objective (100× magnification).

\section{Gross and Surgical Dissection}

Four fresh-frozen cadaveric head and neck specimens were then obtained. They were injected with latex and were imaged with CT scanning to allow for stereotactic image guidance during dissection. The CT scanning protocol was adapted from a thin-cut temporal bone protocol $(0.5 \mathrm{~mm} \times 0.5 \mathrm{~mm}$ slice thickness, $260-\mathrm{mm}$ field of view) and encompassed the midfrontal region down to the level of the C-5 or C-6 vertebral body (specimen dependent).

The Brainlab Skull Reference Base and Brainlab Curve image guidance system were used during surgical dissections. Preoperative planning was performed using the iPlan CMF virtual planning system and imported into the Curve station. The Brainlab Skull Reference Base was affixed to the frontal bone of each specimen using KLS Martin Cross-Drive screws. The specimens were then registered to their preoperative CT scan using the Brainlab Curve image guidance system. Preoperative planning was performed using the iPlan CMF virtual planning system and was imported into the Curve station. Intraoperative visualization was used throughout the dissections.

\section{Transnasal Odontoidectomy}

Using standard endoscopic instrumentation, the middle and inferior turbinates were lateralized. A posterior septectomy was performed, drilling the inferior septum flush with the hard palate. We did not perform turbinectomy or wide sphenoidotomy. A red rubber catheter was then passed through the left nasal cavity and out the mouth, and it was secured with a hemostat to suspend the soft palate. This allowed easy identification of the plane 

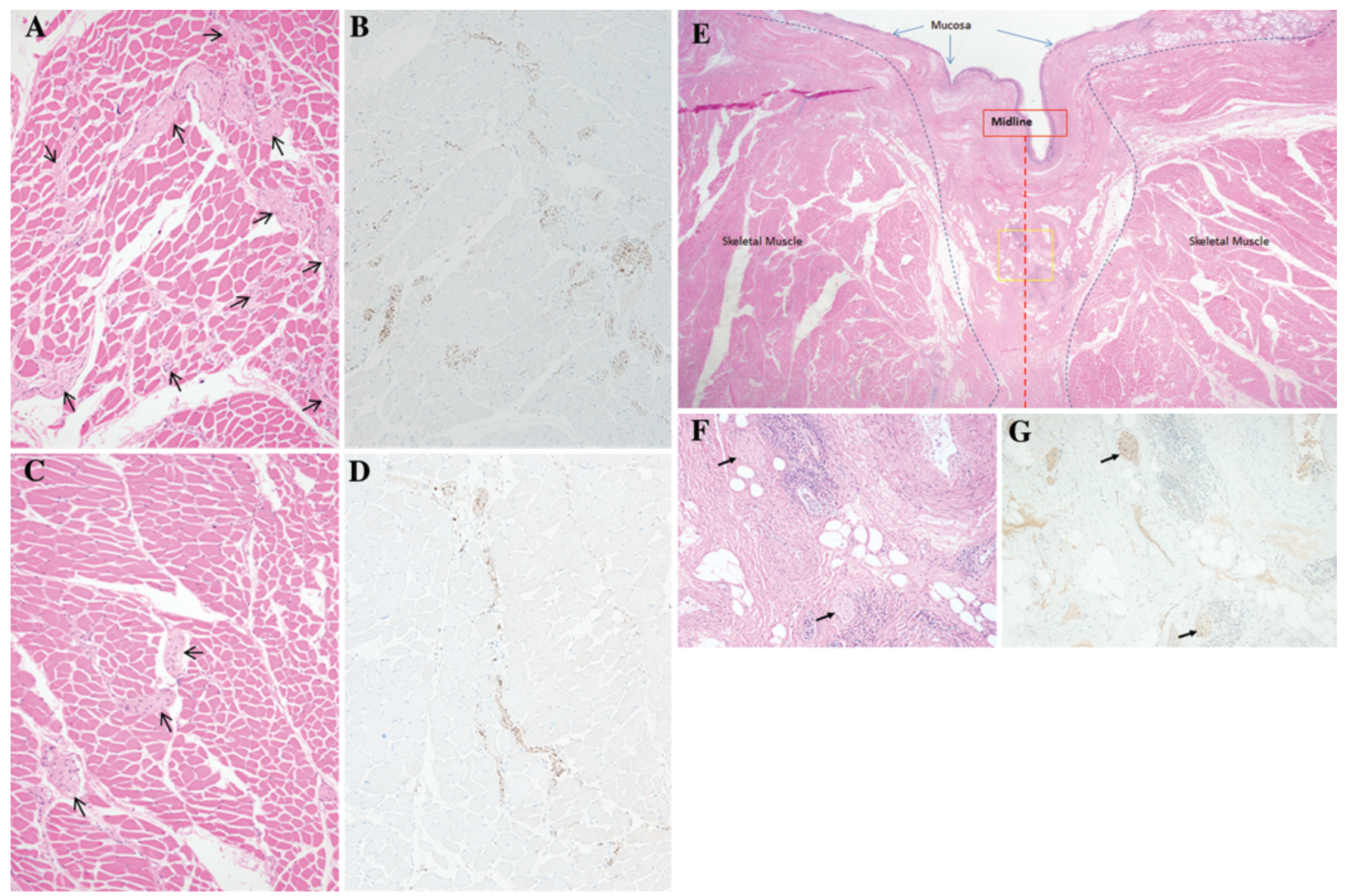

FIG. 2. Photomicrographs demonstrating histology and pharyngeal plexus nerves at the C-2 body and lower clivus. Arrows on the $\mathrm{H} \& \mathrm{E}$ slides indicate nerves. The nerves are stained brown on the $\mathrm{S} 100$ sections. A and B: Representative $\mathrm{H} \& \mathrm{E}$ and corresponding S100 immunohistochemical staining of the posterior pharynx at the vertebral level of the C-2 body (Level 4 from Fig. 1). C and D: Representative H \& E and corresponding S100 immunohistochemical stain of the posterior pharynx at the level of the lower clivus (Level 7 from Fig. 1). Note that the section from the C-2 level clearly has a greater nerve density than the section from the level of the lower clivus. E: Low magnification photomicrograph $(\mathrm{H} \& \mathrm{E})$ of the posterior pharynx. Midline is based off of the raphae, which are both found between the pharyngeal muscles (labeled skeletal muscle). The yellow box bisected by the midline highlights the area seen in photomicrographs $F$ and $G$. F and G: Medium magnification photomicrographs (H \& $E[F]$ and S100 [G]) showing 2 nerves identified within the midline. Original magnification $\times 100(A-D, F$, and $G) ; \times 12.5(E)$.

connecting the inferior aspects of Rosenmüller's fossa bilaterally, approximating the functional division between the oropharynx and nasopharynx, referred to herein as the palatal plane (Fig. 3A). The lymphoid tissue of the nasopharynx was resected, and a linear incision was carried inferiorly. We elevated paraspinal musculature and identified the arch of C-1 and the odontoid process. The arch was then removed, and the odontoid process was resected to its base.

\section{Transoral Odontoidectomy}

We used standard transoral skull base instrumentation and a combination of microscopy and endoscopy for visualization. A Crowe-Davis mouth gag was used, and the soft palate was retracted using 2 red rubber catheters through the nose and was affixed laterally with a hemostat. Using a combination of palpation and image guidance, we identified the arch of C-1 and made our incision vertically in the midline from the superior to inferior extent of the arch of C-1 (Fig. 3B). Dissection was carried laterally to elevate the paraspinal muscles and expose $\mathrm{C}-1$. We then resected the arch and the odontoid process to its base.

\section{Lateral Neck Dissection}

We then performed a lateral neck dissection. We made a curvilinear incision from the mastoid tip to midline, approximately 2 finger breadths below the mandible. Subplatysmal skin flaps were elevated. We then identified the lateral neurovasculature, specifically CNs IX, X, XI, and XII, the sympathetic chain, the internal jugular vein, and the carotid system. Next, we identified the major pharyngeal branches providing innervation to the pharyngeal plexus from CNs IX and $\mathrm{X}$ and the sympathetic chain. We attempted to trace these into the musculature using surgical microscopy.

\section{Posterior Approach/Disarticulated Gross Dissection}

Using image guidance, we placed a single surgical clip at the superior extent of the nasopharyngeal muco- 


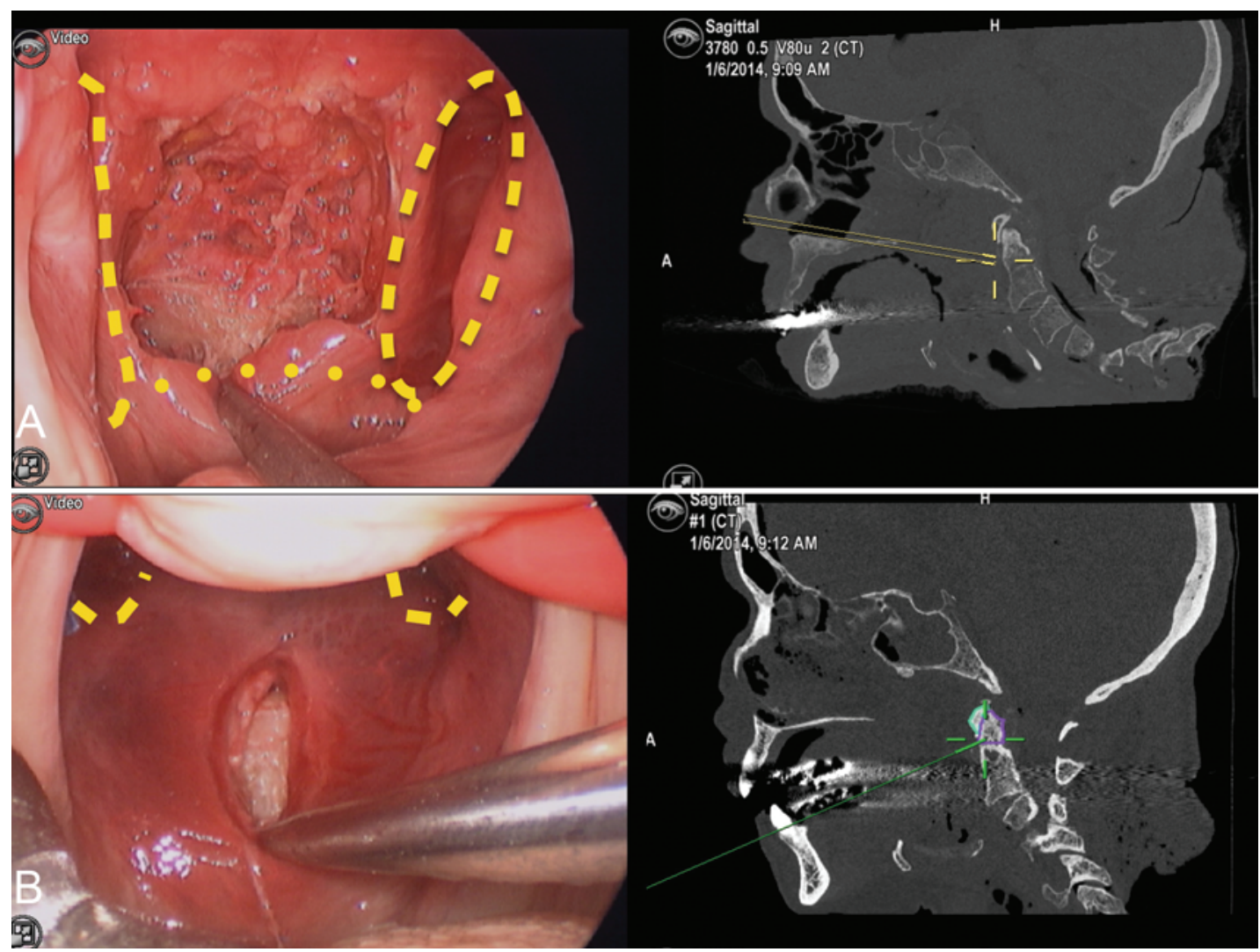

FIG. 3. Intraoperative images and CT scans depicting the approaches for TN and TO odontoidectomies. A: Transnasal odontoidectomy. Endoscopic image (left) depicting the surgical approach after the nasopharyngeal lymphoid tissue has been removed. The dashed lines represent Rosenmüller's fossa and the dotted line represents the palatal plane. The sagittal CT scan (right) demonstrates the inferior access via the TN approach. B: Transoral odontoidectomy. The endoscopic view (left) demonstrates the surgical approach after the soft palate has been suspended. The dashed lines demonstrate the inferior aspect of Rosenmüller's fossa. The sagittal CT scan (right) demonstrates the inferior extent of the transoral incision.

sal flap and a suture at the inferior extent, just above the palatal plane (Fig. 4). An abbreviated TO procedure was performed to identify bony and soft-tissue landmarks at the superior and inferior extent of our TO approach (described above). The suture at the inferior extent of the TN approach served as the superior marker for the TO approach. The inferior extent of the TO approach was marked with a second stitch (Fig. 4).

The specimen was then positioned prone, and the occiput and cervical spine were exposed through a linear incision and subperiosteal muscle dissection. Horizontal incisions were made from the inion to the mastoids, and the dissection was carried laterally and then circumferentially to the cervical spine to reflect the prevertebral fascia (alar and buccopharyngeal) forward. A large handsaw was used to disarticulate $\mathrm{C}-1$ from the condyles and the lower cervical spine from the torso. The cervical spine was dissected free and discarded. The prevertebral fascia was elevated to expose pharyngeal constrictors and their neurovasculature (Fig. 4). We then identified the lateral neurovasculature, specifically CNs VII, IX, X, XI, and XII; the sympathetic chain; the internal jugular vein; and the carotid system. Next, we identified the primary pharyngeal branches providing innervation to the pharyngeal plexus from CNs IX and $\mathrm{X}$ and the sympathetic chain. We attempted to trace these into the musculature using microscopy.

\section{Results}

\section{Histology}

As described above and in Fig. 1, the pharyngeal blocks were sectioned according to level, and nerve densities were calculated. The mean \pm SEM of triplicate readings were as follows: lower C-3 $\left(138.7 \pm 69.6\right.$ nerves $\left./ \mathrm{cm}^{3}\right)$, upper C-3 $\left(164.0 \pm 80.2\right.$ nerves $\left./ \mathrm{cm}^{3}\right)$, lower C-2 (180.3 \pm 59.8 nerves $\left./ \mathrm{cm}^{3}\right)$, body C-1 $\left(179.5 \pm 14.9\right.$ nerves $\left./ \mathrm{cm}^{3}\right)$, lower C-1 $\left(177.0 \pm 26.0\right.$ nerves $\left./ \mathrm{cm}^{3}\right)$, upper C-1 $(108.3 \pm$ 12.2 nerves $\left./ \mathrm{cm}^{3}\right)$, lower clivus $\left(73 \pm 26.6\right.$ nerves $\left./ \mathrm{cm}^{3}\right)$, and midclivus $\left(45 \pm 27.3\right.$ nerves $\left./ \mathrm{cm}^{3}\right)$. ANOVA revealed that the lower and midclivus, both above the palatal plane, had a significantly lower density of nerves compared with all other locations $(\mathrm{p}<0.05)$. We further averaged nerve counts below the hard palate $\left(178.8 \pm 75.4\right.$ nerves $\left./ \mathrm{cm}^{3}\right)$, compared with above the hard palate $(75.4 \pm 32.1$ nerves/ $\mathrm{cm}^{3}$ ), and found that the difference between these 2 subsites was significant $(\mathrm{p}<0.05)$, with a higher density noted below the palatal plane within the oropharynx (Fig. 5).

\section{Gross and Surgical Dissections}

All 3 surgical approaches offered access to the anterior arch of C-1 and the odontoid process. Stereotactic imaging and preoperative surgical planning software fa- 

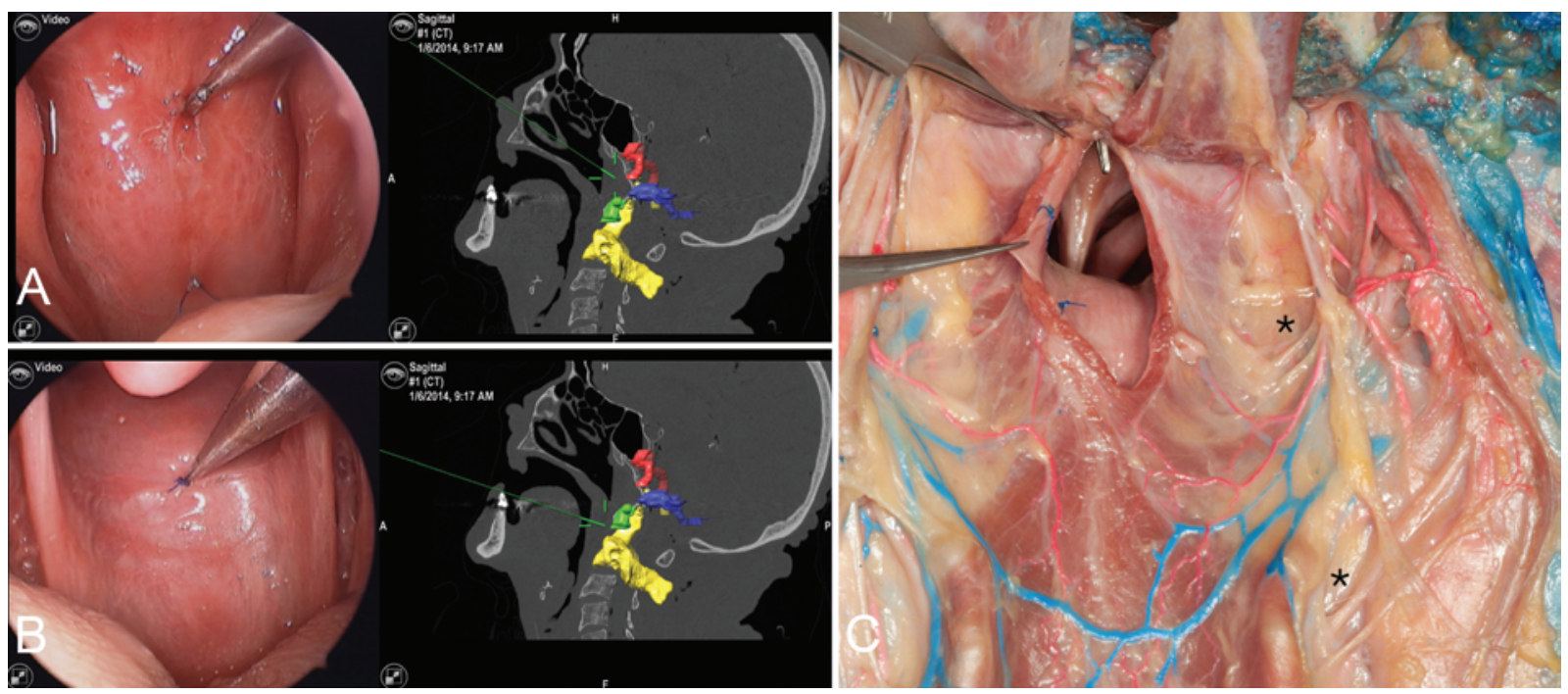

FIG. 4. Posterior gross dissection demonstrating gross anatomy of the pharyngeal constrictors and laterally based neurovasculature and the relationship with the TN and TO surgical approaches to the odontoid process. A: Transnasal odontoidectomy. The endoscopic view (left) demonstrates a clip being placed at the superior extent of the transnasal surgical incision. A suture can be seen inferiorly that marks the inferior extent of this incision. Note it is above the palatal line. The sagittal CT scan (right) again demonstrates the superior access with image guidance. The structures outlined include the arch of C-1 (green) the internal carotid arteries (red), C-2/odontoid (yellow), and jugular vein (blue). B: Transoral odontoidectomy. The endoscopic view (left) demonstrates a suture at the inferior extent of the dissection (the suture seen in the endoscopic view for the transnasal approach was used as the superior extent of the TO approach and cannot be seen in this photograph). The sagittal CT scan (right) demonstrates the inferior extent of the TO incision using image guidance. Outlined structures are identical to those seen in panel A. C: Posterior anatomical dissection of the pharynx highlighting the laterally based primary pharyngeal nerves (asterisks), vasculature (seen with red and blue latex dye), and the pharyngotomy used for the TN and TO approaches. The clip seen superiorly marks the superior extent of the TN approach. The middle suture marks the junction of the inferior extent of the TN approach and the superior extent of the TO approach. The second suture marks the inferior extent of the TO approach.

cilitated analysis of surgical corridors, surgical trajectories, and correlation of surgical landmarks with gross and histopathological dissections.

\section{Lateral and Posterior Dissections}

The major contributions, or pharyngeal branches, of the pharyngeal plexus were identified on gross dissection. As expected, these arose laterally from CNs IX and X as well as the sympathetic chain before running medi- ally to anastomose in the pharyngeal musculature. Nerve branches quickly became indistinguishable from musculature as they approached midline.

\section{Discussion}

One of the most common complications of anterior cervical spine surgery is postoperative dysphagia. ${ }^{2}$ While many authors consider postoperative dysphagia a complication, others simply consider it an unavoidable se-
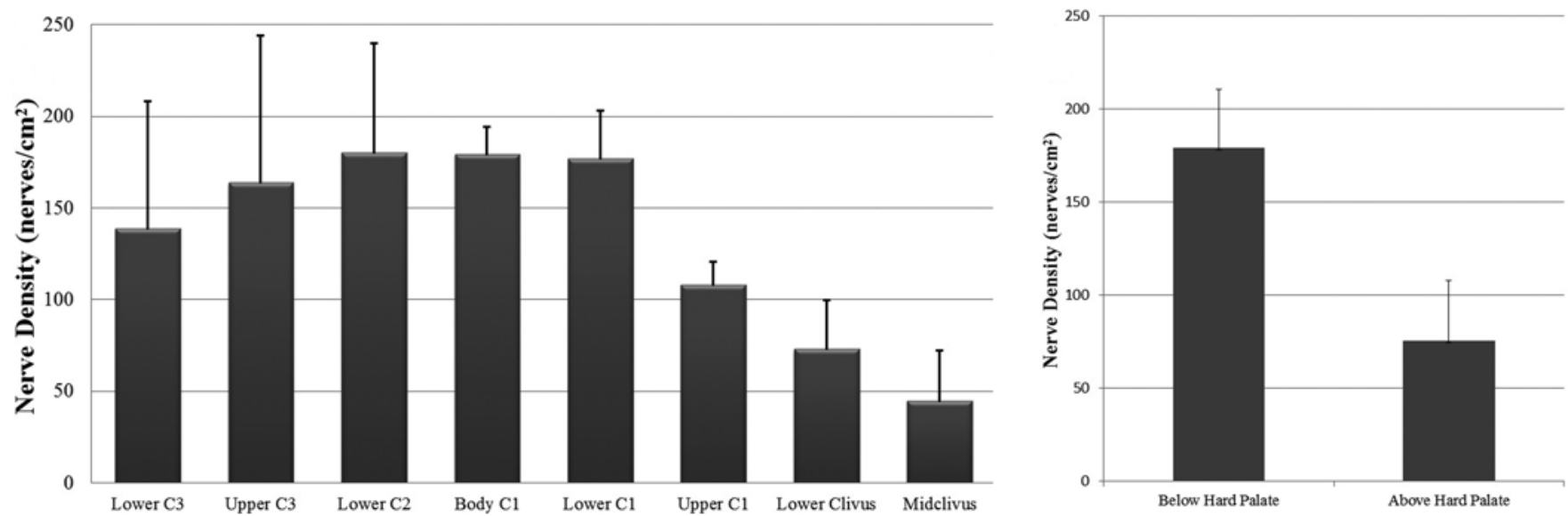

FIG. 5. Nerve densities identified within the pharynx. Left: Nerve densities found in each sectioned level of the 3 specimens processed for histological assessment (see Fig. 1) based on S100 staining. Right: Relative nerve densities identified either above or below the hard palate in these 3 specimens. Statistically significant lower nerve density found above the palatal plane $(p<0.05)$. 
quela of anterior cervical spine surgery. ${ }^{2}$ Despite the fact that postoperative dysphagia is generally transient $(<6$ months), it can result in malnutrition, aspiration, dehydration, and pneumonia, as well as social isolation, depression, and anxiety. ${ }^{11}$ Although there may be many overlapping factors, including postoperative edema, preoperative debility, and neuromuscular injury, evaluating how the surgical anatomy of each approach relates to the neuromuscular anatomy and associated physiology of swallowing is imperative to understanding the risk of postoperative dysphagia.

Swallowing is a complex skill that is dependent on the function of more than 30 muscles, bilateral central nervous system control, and a dense peripheral intramuscular neural plexus. ${ }^{1,2}$ Innervation is provided peripherally by the pharyngeal plexus, which is made up of both sensory and motor nerves. The sensory innervation of the pharynx arises predominantly from the pharyngeal branches of the glossopharyngeal (CN IX) and vagus (CN X) nerves and the internal branch of the superior laryngeal nerve $(\mathrm{CN}$ $\mathrm{X}) .^{23}$ The pharyngeal plexus also provides motor innervation to 2 histochemically defined muscle layers: 1) the fast outer layer and 2) the slow inner layer. ${ }^{22}$ Motor innervation to these 2 layers arises also from the pharyngeal branches of CN IX, CN X, and sympathetic nerves from the superior or cervical sympathetic ganglion. Inferiorly, the internal branch of the superior laryngeal nerve and the recurrent laryngeal nerves also contribute to the pharyngeal plexus and provide motor innervation to the inferior constrictors and the cricopharyngeus muscle. ${ }^{22}$

The primary pharyngeal motor nerves enter the musculature laterally and are distributed to the constrictors in a segmental fashion, giving off secondary and tertiary branches that terminate in a dense network of nerve terminals in the midline. ${ }^{22}$ The primary branches were easily identified in our study and could be seen coursing medially with the vasculature to enter the pharyngeal musculature (Fig. 4). They quickly anastomosed within the musculature and mucosa. In fact, it was impossible during our dissections to identify any neural elements in the midline based on gross dissection alone.

It has traditionally been taught that a midline pharyngotomy through the avascular pharyngeal raphe would result in less injury to the pharyngeal plexus than laterally based or U-shaped incisions. However, this has not been thoroughly investigated in the literature. Interestingly, de Carlos et al. identified numerous and varied sensory corpuscles/mechanoreceptors in the pharynx, including within the midline pharyngeal raphe, which likely detect mechanical changes in the upper aerodigestive tract and contribute to efficient swallowing. ${ }^{8}$ We also demonstrated that there is not only a midline presence of these neuronal elements (Fig. 2), but a significant reduction in the density of nerves found within the posterior nasopharyngeal wall compared with the posterior oropharyngeal wall (Fig. 5). This finding, although circumstantial, may be a contributor to the clinically observed improvement in swallowing outcomes after TN procedures.

The TN approach has recently been touted as a surgical approach to the odontoid process that results in a relatively low risk of postoperative dysphagia. In this study, we felt that the TN approach resulted in the least potential injury to the pharyngeal plexus. The midline approach limited injury to the laterally based primary pharyngeal plexus nerves, and by keeping the incision above the palatal plane, we avoided injury of the pharyngeal plexus associated with the oropharyngeal wall. However, this approach was the most time consuming and potentially requires 2 surgeons, or a 4 -handed technique.

To minimize postoperative dysphagia, there must be an inferior limit to the surgical incision for TN odontoidectomy that maximizes bony exposure while minimizing injury to velopharyngeal and oropharyngeal structures. We defined this limit as the palatal plane. This line can be drawn between the inferior aspect of Rosenmüller's fossa bilaterally and approximates the upper limit of the superior pharyngeal constrictor at the level of the palatopharyngeal sphincter (Fig. 3A) ${ }^{5,14}$ The finding that neural density was significantly higher below this line within the oropharynx (Fig. 5) further supports the hypothesis that the TN approach minimizes injury to the pharyngeal plexus and therefore may decrease the risk of postoperative dysphagia.

The body of literature reporting swallowing outcomes for patients undergoing TN odontoidectomy is limited, but it is growing. We identified 15 studies discussing 52 patients (Table 1).

Of these, only 48 patients had been swallowing preoperatively. When looking at this population, 83\% (40 of 48) were swallowing by Day 3 and $92 \%$ (44 of 48) were swallowing by Day 7 . Three of the 4 remaining patients had a percutaneous endoscopic gastrostomy tube placed postoperatively, which was felt by Nayak et al. to be reflective of their preoperative pharyngeal morbidity. ${ }^{24}$ The final patient required an emergency tracheostomy immediately postoperatively for oversedation and was decannulated on postoperative Day $6 .^{12}$ At 1 month his swallowing had normalized, but there is no mention of whether oral or enteral feeding was used during that 1 month.

These data suggest that at 1 week following surgery, only $8 \%$ of patients had significant dysphagia requiring enteral feeding following the TN approach. This is significantly less than the $50 \%-60 \%$ rate reported for general anterior cervical spine injury $y^{3,20}$ and the $90 \%-100 \%$ risk following TO approaches..$^{26,28}$ These data are variable, difficult to compare, include patients with a variety of neurological comorbidities, and are largely descriptive. However, they provide an initial analysis of postoperative dysphagia following TN odontoidectomy that can be used as a baseline to compare with other approaches.

In our study, the TO approach avoided injuring the surgically visible primary pharyngeal plexus nerves laterally, but it did require transection of the posterior oropharyngeal wall, which has the highest density of pharyngeal plexus nerve fibers. In addition, the pharyngeal musculature, which is intimately associated with oropharyngeal swallowing, must be transected and manipulated. Finally, the incision must be placed intraorally, heightening the risk associated with increased contact with saliva and oral flora. However, the TO approach provided the most direct access and was accomplished in the shortest amount of time. 
Transnasal endoscopic odontoidectomy and postoperative dysphagia

TABLE 1: Literature review of swallowing outcomes for patients following transnasal endoscopic odontoidectomy*

\begin{tabular}{|c|c|c|c|c|c|}
\hline Authors \& Year & $\begin{array}{c}\text { No. of } \\
\text { Patients }\end{array}$ & $\begin{array}{c}\text { Mean Age in Yrs } \\
\text { (range) }\end{array}$ & $\begin{array}{l}\text { No. Requiring PEG } \\
\text { Tube Feeding Postop }\end{array}$ & $\begin{array}{l}\text { No. Requiring } \\
\text { NGT >72 Hrs }\end{array}$ & $\begin{array}{c}\text { No. Swallowing by } \\
72 \text { Hrs Postop }\end{array}$ \\
\hline Schwartz et al., 2014 & 9 & $54.8(7-77)$ & 0 & 0 & 9 \\
\hline Choudhri et al., 2014 & 5 & $67(55-87)$ & 0 & 0 & 5 \\
\hline Mazzatenta et al., 2014 & 5 & $30(11-64)$ & $1 \dagger$ & 0 & 4 \\
\hline lacoangeli et al., 2013 & 3 & $76.6(70-80)$ & 0 & 0 & 3 \\
\hline Hickman et al., 2013 & 2 & 11,12 & $2 \dagger$ & 0 & 0 \\
\hline Yu et al., 2013 & 3 & 51 & 0 & 0 & 3 \\
\hline Gladi et al., 2012 & 4 & $74(69-80)$ & 0 & $2 \ddagger$ & 2 \\
\hline Cornelius et al., 2011 & 1 & 57 & 0 & 0 & 1 \\
\hline Hankinson et al., 2010 & $1(2) \S$ & 15 & 0 & 1 & 0 \\
\hline Gempt et al., 2011 & 3 & $64(52-77)$ & 0 & $3 \pi$ & 0 \\
\hline Leng et al., 2009 & 1 & 50 & 0 & 0 & 1 \\
\hline Laufer et al., 2008 & 1 & 25 & 0 & 0 & 1 \\
\hline Wu et al., 2008 & 3 & $44(33-59)$ & 0 & 0 & 3 \\
\hline Nayak et al., 2007 & 9 & 76 & 3 & 0 & 6 \\
\hline Kassam et al., $2005^{16}$ & 1 & 68 & 0 & 0 & 1 \\
\hline Kassam et al., $2005^{17}$ & 1 & 73 & 0 & 0 & 1 \\
\hline total & 52 & & 6 & 6 & 40 \\
\hline
\end{tabular}

* NGT = nasogastric tube; $P E G$ = percutaneous endoscopic gastrostomy.

$\dagger$ These patients were dependent on a percutaneous endoscopic gastrostomy tube preoperatively.

$\ddagger$ Both patients resumed an oral diet by Day 5 .

$\S$ One patient underwent an emergency tracheostomy and underwent decannulation on postoperative Day 6 . At 1 month his swallowing had normalized, but there is no mention of whether oral or enteral feeding was used during that 1 month. There is no swallowing information for the second patient other than that the patient was asymptomatic at 18 months.

If One patient was dependent on a nasogastric tube preoperatively for 3 months. All were preemptively fed via gastric tube for 7 days. The patient dependent on a nasogastric tube preoperatively required enteral feeding for 3 weeks postoperatively. The other 2 patients returned to oral intake at 7 days.

While the TO approach is still considered the gold standard for anterior decompression of the odontoid process, it is now thought to have the highest risk of postoperative dysphagia. In a study by Yadav et al. in 2013, which examined 34 patients who underwent $\mathrm{TO}$, the authors stated, "most patients experienced some swallowing difficulties for 2-3 weeks." ${ }^{28}$ In 2011, Van Gompel et al. reported their experience in a population of patients undergoing TO for cystic deterioration of the C1-2 articulation. ${ }^{26}$ In that study, all but 1 patient required enteral tube feeding for more than 72 hours after surgery (mean 14 days, range 6-30 days). One patient returned to an oral diet after 48 hours, and a final patient required a temporary percutaneous gastrostomy tube. This represents a $90 \%-100 \%$ risk of significant postoperative dysphagia.

\section{Conclusions}

By limiting the $\mathrm{TN}$ incision to above the palatal plane, the surgeon avoids laterally based primary pharyngeal plexus nerves and the high-density neural plexus found in the oropharyngeal wall. This may explain the decreased incidence of postoperative dysphagia seen in TN approaches. While this study provides initial evidence that there may be an anatomical and physiological reason for the decreased risk of postoperative dysphagia after TN compared with the TO approach, further clinical study is warranted.

\section{Disclosure}

An internal Departmental Grant was awarded through the department of neurosurgery for this work. Brainlab donated the Brainlab Curve image guidance system for use during this investigation.

Author contributions to the study and manuscript preparation include the following. Conception and design: Van Gompel, Van Abel, Mallory, Jentoft. Acquisition of data: Van Gompel, Van Abel, Mallory, Kasperbauer, Jentoft. Analysis and interpretation of data: Van Gompel, Van Abel, Jentoft. Drafting the article: Van Gompel, Van Abel, Mallory. Critically revising the article: Van Gompel, Moore, O'Brien, Olsen, Jentoft. Reviewed submitted version of manuscript: Van Gompel, Mallory, Kasperbauer, Moore, Price, O'Brien, Olsen, Krauss, Clarke, Jentoft. Statistical analysis: Van Gompel.

\section{References}

1. Abe S, Fukuda M, Yamane S, Saka H, Katori Y, RodríguezVázquez JF, et al: Fetal anatomy of the upper pharyngeal muscles with special reference to the nerve supply: is it an enteric plexus or simply an intramuscular nerve? Anat Cell Biol 46:141-148, 2013

2. Anderson KK, Arnold PM: Oropharyngeal dysphagia after anterior cervical spine surgery: a review. Global Spine J 3: 273-286, 2013 
3. Bazaz R, Lee MJ, Yoo JU: Incidence of dysphagia after anterior cervical spine surgery: a prospective study. Spine (Phila Pa 1976) 27:2453-2458, 2002

4. Belafsky PC, Mouadeb DA, Rees CJ, Pryor JC, Postma GN, Allen J, et al: Validity and reliability of the Eating Assessment Tool (EAT-10). Ann Otol Rhinol Laryngol 117:919-924, 2008

5. Cho JH, Kim JK, Lee HY, Yoon JH: Surgical anatomy of human soft palate. Laryngoscope 123:2900-2904, 2013

6. Choudhri O, Mindea SA, Feroze A, Soudry E, Chang SD, Nayak JV: Experience with intraoperative navigation and imaging during endoscopic transnasal spinal approaches to the foramen magnum and odontoid. Neurosurg Focus 36(3):E4, 2014

7. Cornelius JF, Kania R, Bostelmann R, Herman P, George B: Transnasal endoscopic odontoidectomy after occipito-cervical fusion during the same operative setting-technical note. Neurosurg Rev 34:115-121, 2011

8. de Carlos F, Cobo J, Macías E, Feito J, Cobo T, Calavia MG, et al: The sensory innervation of the human pharynx: searching for mechanoreceptors. Anat Rec (Hoboken) 296:1735-1746, 2013

9. Gempt J, Lehmberg J, Grams AE, Berends L, Meyer B, Stoffel M: Endoscopic transnasal resection of the odontoid: case series and clinical course. Eur Spine J 20:661-666, 2011

10. Gladi M, Iacoangeli M, Specchia N, Re M, Dobran M, Alvaro L, et al: Endoscopic transnasal odontoid resection to decompress the bulbo-medullary junction: a reliable anterior minimally invasive technique without posterior fusion. Eur Spine J 21 (Suppl 1):S55-S60, 2012

11. Goldsmith TA, Holman AS, Nunn D: Videofluoroscopic evaluation of oropharyngeal swallowing, in Som PM, Curtin HD (eds): Head and Neck Imaging, ed 5. St. Louis: Mosby, 2011, Vol 2, pp 2085-2113

12. Hankinson TC, Grunstein E, Gardner P, Spinks TJ, Anderson RCE: Transnasal odontoid resection followed by posterior decompression and occipitocervical fusion in children with Chiari malformation Type I and ventral brainstem compression. Report of 2 cases. J Neurosurg Pediatr 5:549-553, 2010

13. Hickman ZL, McDowell MM, Barton SM, Sussman ES, Grunstein E, Anderson RCE: Transnasal endoscopic approach to the pediatric craniovertebral junction and rostral cervical spine: case series and literature review. Neurosurg Focus 35(2):E14, 2013

14. Hollinshead HW: The pharynx and larynx, in: Anatomy for Surgeons: Volume 1. The Head and Neck, ed 3. Philadelphia: Harper and Row, 1982, pp 389-442

15. Iacoangeli M, Gladi M, Alvaro L, Di Rienzo A, Specchia N, Scerrati M: Endoscopic endonasal odontoidectomy with anterior $\mathrm{C} 1$ arch preservation in elderly patients affected by rheumatoid arthritis. Spine J 13:542-548, 2013

16. Kassam AB, Abla A, Snyderman C, Carrau R, Spiro R: An endoscopic transnasal odontoidectomy to treat cervicomedullary compression with basilar invagination. Op Tech Neurosurg 8:198-204, 2005

17. Kassam AB, Snyderman C, Gardner P, Carrau R, Spiro R: The expanded endonasal approach: a fully endoscopic transnasal approach and resection of the odontoid process: technical case report. Neurosurgery 57 (1 Suppl):E213, 2005

18. Laufer I, Greenfield JP, Anand VK, Härtl R, Schwartz TH:
Endonasal endoscopic resection of the odontoid process in a nonachondroplastic dwarf with juvenile rheumatoid arthritis: feasibility of the approach and utility of the intraoperative Iso-C three-dimensional navigation. Case report. J Neurosurg Spine 8:376-380, 2008

19. Leng LZ, Anand VK, Härtl R, Schwartz TH: Endonasal endoscopic resection of an os odontoideum to decompress the cervicomedullary junction: a minimal access surgical technique. Spine (Phila Pa 1976) 34:E139-E143, 2009

20. Leonard R, Belafsky P: Dysphagia following cervical spine surgery with anterior instrumentation: evidence from fluoroscopic swallow studies. Spine (Phila Pa 1976) 36:2217-2223, 2011

21. Mazzatenta D, Zoli M, Mascari C, Pasquini E, Frank G: Endoscopic endonasal odontoidectomy: clinical series. Spine (Phila Pa 1976) 39:846-853, 2014

22. Mu L, Sanders I: Neuromuscular specializations within human pharyngeal constrictor muscles. Ann Otol Rhinol Laryngol 116:604-617, 2007

23. Mu L, Sanders I: Sensory nerve supply of the human oro- and laryngopharynx: a preliminary study. Anat Rec 258:406-420, 2000

24. Nayak JV, Gardner PA, Vescan AD, Carrau RL, Kassam AB, Snyderman CH: Experience with the expanded endonasal approach for resection of the odontoid process in rheumatoid disease. Am J Rhinol 21:601-606, 2007

25. Schwartz TH, Goldschlager T, Hartl R, Greenfield JP, Anand VK: The endoscopic endonasal approach to the odontoid and its impact on early extubation and feeding. J Neurol Surg B Skull Base 75 (Suppl 1):A049, 2014 (Abstract)

26. Van Gompel JJ, Morris JM, Kasperbauer JL, Graner DE, Krauss WE: Cystic deterioration of the C1-2 articulation: clinical implications and treatment outcomes. Clinical article. J Neurosurg Spine 14:437-443, 2011

27. Wu JC, Huang WC, Cheng H, Liang ML, Ho CY, Wong TT, et al: Endoscopic transnasal transclival odontoidectomy: a new approach to decompression: technical case report. Neurosurgery 63 (1 Suppl 1):ONSE92-ONSE94, 2008

28. Yadav YR, Madhariya SN, Parihar VS, Namdev H, Bhatele PR: Endoscopic transoral excision of odontoid process in irreducible atlantoaxial dislocation: our experience of 34 patients. J Neurol Surg A Cent Eur Neurosurg 74:162-167, 2013

29. Yu Y, Wang X, Zhang X, Hu F, Gu Y, Xie T, et al: Endoscopic transnasal odontoidectomy to treat basilar invagination with congenital osseous malformations. Eur Spine J 22:1127-1136, 2013

30. Zeng JH, Zhong ZM, Chen JT: Early dysphagia complicating anterior cervical spine surgery: incidence and risk factors. Arch Orthop Trauma Surg 133:1067-1071, 2013

Manuscript submitted June 15, 2014.

Accepted July 15, 2014.

Please include this information when citing this paper: DOI: 10.3171/2014.7.FOCUS14338.

Address correspondence to: Jamie J. Van Gompel, M.D., Department of Neurosurgery, Mayo Clinic, 200 First St. SW, Rochester, MN 55905. email: vangompel.jamie@mayo.edu. 